C2008 IEEE. Personal use of this material is permitted. However, permission to reprint/republish this material for advertising or promotional purposes or for creating new collective works for resale or redistribution to servers or lists, or to reuse any copyrighted component of this work in other works must be obtained from the IEEE. 


\title{
Adaptive Setting of OLTC to Improve Power Transfer Capability of Power Systems
}

\author{
A.Abu-Siada1* S. Islam1 and E.A. Mohamed2 \\ 1Department of Electrical and Computer Engineering, Curtin University of Technology, WA6845, Australia \\ 2 Electrical power and Machine Department, Ain Shams University, Cairo, Egypt \\ *E-mail: a.abusiada@curtin.edu.au
}

\begin{abstract}
On load tap changing transformer (OLTC) has become a vital link in modern power systems. It acts to maintain the load bus voltage within its permissible limits despite any load changes. This paper discusses the effect of different static loads namely; constant power (CP), constant current (CI) and constant impedance $(\mathrm{CZ})$ on the maximum power transfer limit from the generation to the load centre through the OLTC branch and in turn on the static stability margin of power systems. Then the paper introduces a novel approach for the on-line determination of the OLTC settings in order to improve the power transfer capability of transmission systems. The proposed approach is tested based on the simulation of a six-bus power system. Numerical results prove that the setting of transformer OLTC in terms of the load model has a major effect on the maximum power transfer in power systems. The adaptive settings of OLTC improve the power transfer capability according to the system operating condition.
\end{abstract}

Index Term--OLTC, static load, power transfer limit, stability margin, ANN

\section{INTRODUCTION}

$\mathrm{M}$ odern power systems are being stressed with the continuous growth of load requirements. Power system operator should ensure the quality and reliability of supply to the loads by maintaining the load bus voltages within their permissible limits. The power transfer from generators to load centers affects load bus voltages. In addition to restoring the load, OLTC also extend the power transfer ability to the load center [1]. It is well known that the operation of OLTC has a significant influence on voltage stability. Most literature has concentrated on the contribution of OLTC to voltage stability [2]. The power transfer capability is measured by the margin from a base case operating point to the operation-limiting boundary, measured along the load growth trajectory. The static load models have an impact on the power transfer capability from generating station to the load center and thus on the static stability margin of power systems [3]. Therefore, it is important to consider first the effect of different static load models namely; constant power (CP), constant current (CI) and constant impedance (CZ) load models on the power transfer limits to the load centre. Then the paper presents a novel approach for evaluating the on-line optimal settings of transformer OLTC using artificial neural network (ANN) that corresponds to the maximum power transfer to the load and hence to improve the static stability limits.

\section{EfFect of OLTC Setting ON Power Transfer}

Assume the simple power system shown in Fig. 1. The load power is supplied from an infinite bus via OLTC. The load flow equation at load bus is given by:

$\frac{n E-V_{L}}{j n^{2} X_{T}}=\frac{P-j Q}{V_{L}^{4}}+\frac{V_{L}}{-j X_{c}}$

where $E=E+j 0$ is the emf of the equivalent voltage source, $V_{L}=V_{r}+j V_{j}$ is the load bus voltage, $X_{r}=X_{e q}+X_{\nu}$ is the equivalent reactance of power system \& transformer, $\mathrm{P}$ and $\mathrm{Q}$ are the load active and reactive power and $X_{c}$ is the reactance of the shunt capacitor compensated load

\section{A. Constant Power (CP) Load:}

For constant Power load;

$P=c_{r} \quad$ and $\quad Q=c_{q}$

Let $\frac{Q}{P}=\frac{c_{r}}{c_{q}}=K$

Equation (1) can be re-written as below:

$-j \frac{E V_{r}}{n X_{r}}-\frac{E V_{i}}{n X_{r}}+j\left(V_{r}^{2}+V_{i}^{2}\right) \frac{X_{c}-n^{2} X_{r}}{n^{2} X_{r} X_{c}}=P-j K P$

Equating real parts in both sides,

$V_{i}=-\frac{n X_{T}}{E} P$

Equating imaginary parts in both sides and substituting for $V_{i}$ from (3),

$\frac{n^{2} X_{r}^{2}}{E^{2}} P^{2}+\frac{n^{2} K X_{r} X_{c}}{X_{c}-n^{2} X_{r}} P+\left(V_{,}^{2}-\frac{n X_{c} E}{X_{c}-n^{2} X_{r}} V,\right)=0$

Solving for $P$,

$P=\frac{K X_{E}^{2}}{2 X_{\tau}\left(X_{r}-n^{2} X_{\tau}\right)}+\sqrt{\left.\frac{K X E^{2}}{2 X_{r}\left(X_{r}-n^{2} X_{r}\right)}\right)^{2}-\left(\frac{E}{n X_{r}}\right)^{2}\left(V_{r}^{2}-\frac{n X_{r} E}{X_{r}-n^{2} X_{\tau}} V\right)}$

This solution is subject to:

$\left(\frac{K X_{c} E^{2}}{2 X_{T}\left(X_{c}-n^{2} X_{T}\right)}\right)^{2}-\left(\frac{E}{n X_{T}}\right)^{2}\left(V_{r}^{2}-\frac{n X_{c} E}{X_{c}-n^{2} X_{r}} V\right) \succ 0$

and

$n \neq \sqrt{\frac{X_{c}}{X_{r}}}$

From equation (6), one can conclude that there is a limit to the power transfer to the load depends on the degree of compensation, OLTC setting and load bus voltage. Figure 2 shows the effect of OLTC tap ratio on the maximum power transfer for compensated and uncompensated load $\left(\mathrm{X}_{\mathrm{c}}=10 \mathrm{pu}\right.$. and $\mathrm{X}_{\mathrm{c}}=\infty$ respectively). For both cases the maximum power transfer limit is increased by increasing the tap ratio. Once the optimal power is reached $(0.5$ pu. in case of uncompensated load and $0.56 \mathrm{pu}$. in case of compensated load) the power slightly decreases with the increase of tap ratio. It should be noted that for the compensated load, the 
power is dramatically increased when the resonant case is reached $\left(n=\sqrt{\frac{X_{c}}{X_{T}}}=4.472\right.$ in this case $)$

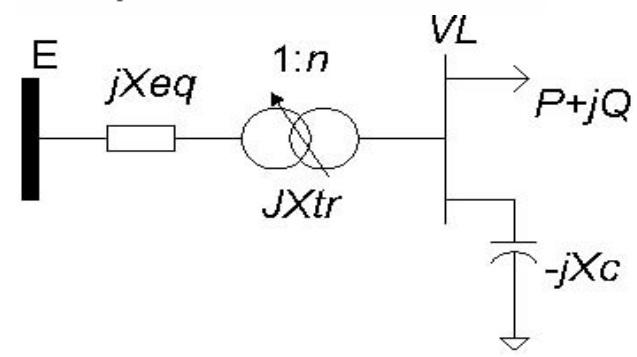

Fig. 1 Simplified power system

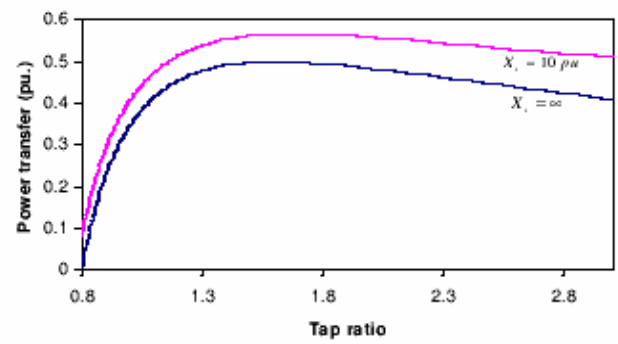

Fig. 2 Effect of OLTC on power transfer to the CP load $(\mathrm{K}=0.75$, XT $=0.5$ pu. $V r=0.8 \mathrm{pu}$.)

Rearranging equation (4) and solving for $\mathrm{Vr}$,

$V=\frac{n X E}{\left.2 X_{c}-n^{2} X_{r}\right)} \pm \sqrt{\left(\frac{n X E}{2\left(X_{c}-n^{2} X_{r}\right)}\right)^{2}-n^{2} X_{r} P\left(\frac{K X}{X_{c}-n^{2} X_{r}}+\frac{X_{\tau}}{E^{2}} P\right)}$

Figure 3 shows the PV curve for the uncompensated load for different tap ratios. The figure shows that the maximum power limit is constant and equal $0.5 \mathrm{pu}$. in all cases.

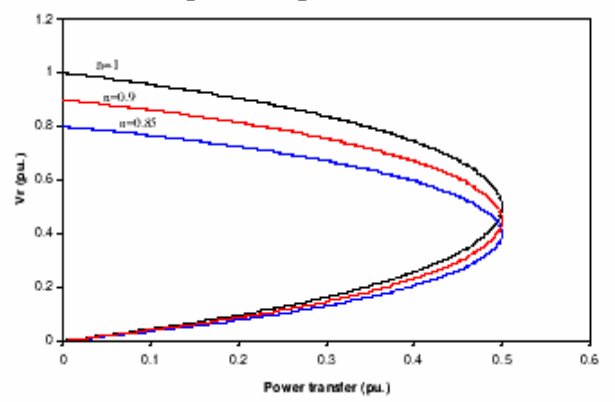

Fig. $3 \mathrm{P}-\mathrm{V}$ curve at different load compensation $\left(X_{c}=\infty\right)$

The PV curves in Figures 4 and 5 show that the maximum power transfer limit to a compensated load can be increased by either increasing the tap ratio of OLTC or by increasing the degree of compensation. The power increase is attributed to the fact that the OLTC tap settings allow the match between the network impedance and the reflected compensated load impedance.

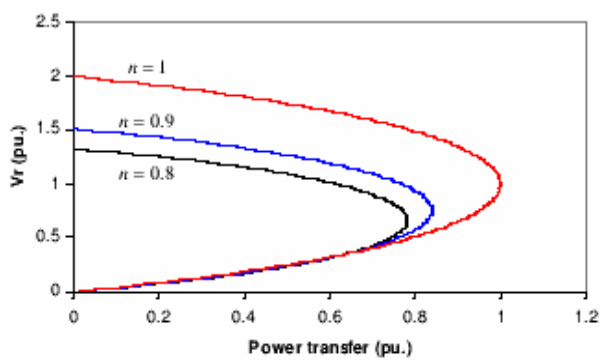

Fig. 4 P-V curve at different load compensation $\left(X_{c}=1 \mathrm{pu}\right)$

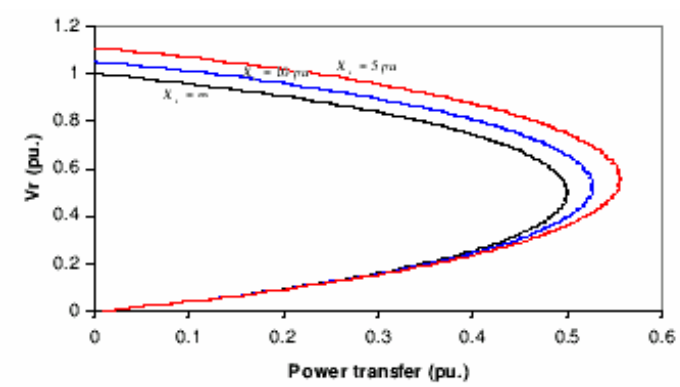

Fig. 5 P-V curve at different load compensation $(n=1)$

B. Constant Current (CI) Load

For constant current load;

$$
P=c_{p}\left|V_{L}\right|, \quad Q=c_{q}\left|V_{L}\right| \quad \frac{Q}{P}=K
$$

Then equation (1) can be re-written as:

$-j \frac{E V_{r}}{n X_{T}}-\frac{E V_{i}}{n X_{T}}+j\left(V_{r}^{2}+V_{i}^{2}\right) \frac{X_{r}-n^{2} X_{T}}{n^{2} X_{T} X_{r}}=c_{r}\left|V_{L}\right|(1-j K)$

Equating real parts in both sides,

$$
\begin{aligned}
& -\frac{E V_{i}}{n X_{T}}=c_{p}\left|V_{L}\right| \\
& \Rightarrow E^{2} V_{i}^{2}=\left(n X_{T} c_{p}\right)^{2}\left(V_{i}^{2}+V_{i}^{2}\right) \\
& \Rightarrow V_{i}= \pm \frac{n X_{T} c_{p}}{\sqrt{E^{2}-\left(n X_{T} c_{p}\right)^{2}}} V_{,}
\end{aligned}
$$

$\left.P=c_{\Gamma} \mid V_{L}\right]=\frac{n c_{r} X_{c}}{X_{c}-n^{2} X_{T}} \sqrt{E^{2}-\left(n X_{T} c_{r}\right)^{2}}-\frac{n^{2} X_{r} X_{T} c_{r}^{2} K}{X_{c}-n^{2} X_{T}}$

This solution is subject to:

$$
\begin{aligned}
& E^{2}-\left(n X_{\tau} c_{\mathrm{p}}\right)^{2}>0 \\
& \Rightarrow n \prec\left|\frac{E}{\mid X_{T} c_{\mathrm{p}}}\right|
\end{aligned}
$$

and

$$
n \neq \sqrt{\frac{X_{c}}{X_{\tau}}}
$$

Figure 6 shows the effect of OLTC tap ratio on the power transfer limit to constant current load. The maximum power transfer limit is increasing with the increase of the tap ratio. When the maximum limit is reached $(0.5 \mathrm{pu}$. in case of uncompensated load and $0.62 \mathrm{pu}$. in case of compensated load), the power is decreasing with the increase of tap ratio. The shunt capacitor increases the power transfer limit and shifts the optimal setting of OLTC to a higher value.

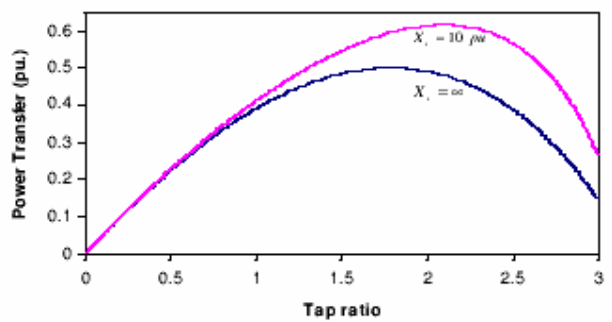

Figure 6 Effect of OLTC on power transfer to the CI load 


\section{Constant Impedance (CZ) Load}

For constant impedance load;

$P=c_{p}\left|V_{L}\right|^{2} \quad Q=c_{q}\left|V_{L}\right|^{2} \quad \frac{Q}{P}=K$

Then equation (1) can be re-written as:

$-j \frac{E V,}{n X_{T}}-\frac{E V_{i}}{n X_{T}}+j\left(V_{r}^{2}+V_{i}^{2}\right) \frac{X_{c}-n^{2} X_{T}}{n^{2} X_{T} X_{c}}=P(1-j K)$

Equating the real parts in both sides,

$V_{i}=-\frac{n X_{T} P}{E}$

Equating imaginary parts,

$V_{r}=\frac{n X_{T}}{E c_{p}}\left(K c_{p}+\frac{X_{c}-n^{2} X_{T}}{n^{2} X_{T} X_{c}}\right) P$

$\left|V_{L}\right|^{2}=V_{r}^{2}+V_{i}^{2}=\left(\frac{n X_{T}}{E c_{r}}\right)^{2}\left(K c_{r}+\frac{X_{r}-n^{2} X_{T}}{n^{2} X_{\tau} X_{r}}\right)^{2} P^{2}+\left(\frac{n X_{T}}{E}\right)^{2} P^{2}=\frac{P}{c_{r}}$

$\Rightarrow P=\frac{1 / c_{p}}{\left(\frac{X_{c}-n^{2} X_{T}}{n c_{p} X_{c} E}+\frac{K n X_{T}}{E}\right)^{2}+\left(\frac{n X_{T}}{E}\right)^{2}}$

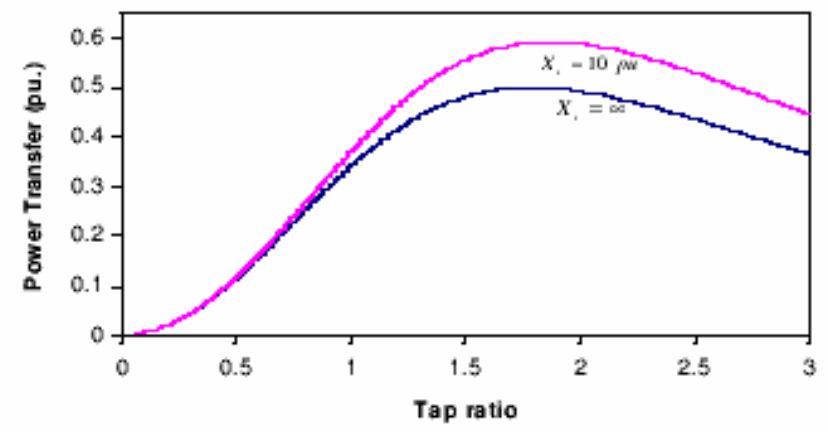

Fig. 7 Effect of OLTC on power transfer to the CZ load

Figure 7 shows the effect of OLTC tap ratio on the power transfer limit to constant impedance load. Again, the power is increasing with the increase of OLTC tap ratio till the optimal power is reached then the power is decreasing with the increase of tap ratio. The optimal power in case of uncompensated load $(\mathrm{Xc}=\infty)$ is $0.5 \mathrm{pu}$. corresponding to 1.72 tap ratio. However, the maximum power for a compensated load $(\mathrm{Xc}=10 \mathrm{pu}$.) is $0.62 \mathrm{pu}$. corresponding to 2.1 tap ratio. It should be noted that in all load models (CP, CI and CZ) the power transfer limit is increased by increasing the degree of compensation i.e. decreasing the value of Xc. When the load power can not be met with the increasing the compensation degree, the power can be increased by increasing the OLTC tap ratio.

\section{Digital Simulation}

Figure 8 shows a one-line diagram of the test system. Thesystem consists of 6-nodes, 7 lines, two transformers OLTC, two generators and four loads [4,5]. The two OLTCs are varied one at a time, during the calculation of the maximum power transfer to the load centres.

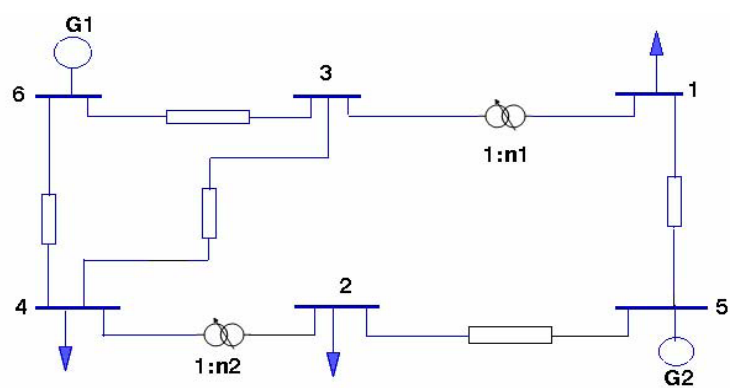

Fig. 8 One line diagram of the system under study Hardware Organization

The results obtained by using the conventional methodology cover a wide range of operating conditions. A sample of the training results is shown in Figs. 9-14. These results are presented to explore the effect of both OLTC settings and the load model on the maximum power transfer. Figures 9-11 show the relation between the power transfer and the transformer tap ratio $\mathrm{n}$ at the three load buses $(1,2 \& 4)$ with the loading condition $\mathrm{PL}=\mathrm{QL}=0.35$ pu. $(\mathrm{pf}=0.707 \mathrm{lag})$, for the three different load models (CP, CI \& CZ). From these figures, it can be seen that the optimum value of $n$ and the corresponding maximum power transfer are different for the three load models. Figures 12-14 present similar results at another loading condition $\mathrm{PL}=0.8 \mathrm{pu}$. and $\mathrm{QL}=0.13 \mathrm{pu}$. (pf $=0.987 \mathrm{lag}$ ). It can be seen from these figures that the maximum power transfer limit is higher at this operating condition than the previous case and the OLTC setting $\mathrm{n} 1$ has a strong effect on the load at bus \#1 which is similar to the effect of $\mathrm{n} 2$ on the load at bus \#2. On the other hand, the OLTC setting $\mathrm{n} 2$ has a unique correlation and effect on the load at bus \#3. These figures prove that the power transfer limit is affected by the OLTC setting for all load models.

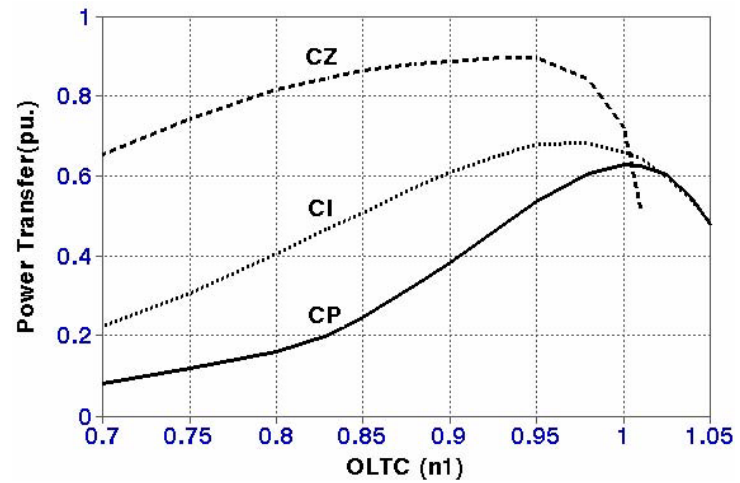

Fig. 9 Effect of OLTC (n1) on Power transfer to load 1

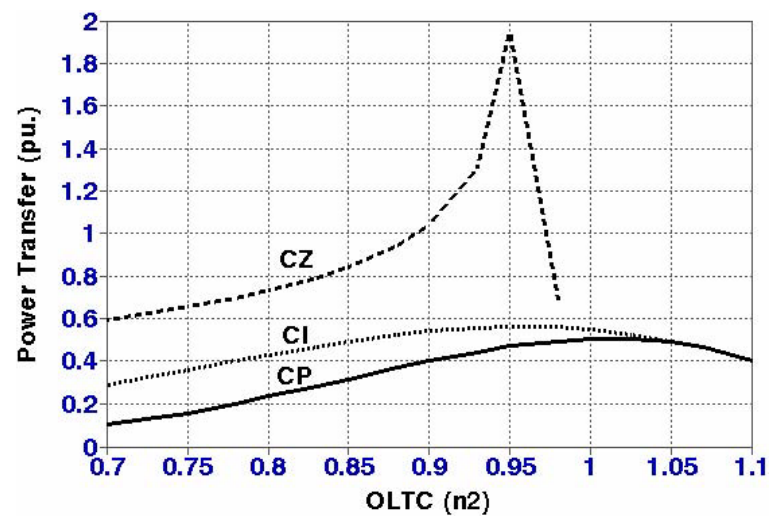

Fig. 10 Effect of OLTC (n2) on Power transfer to load 2 


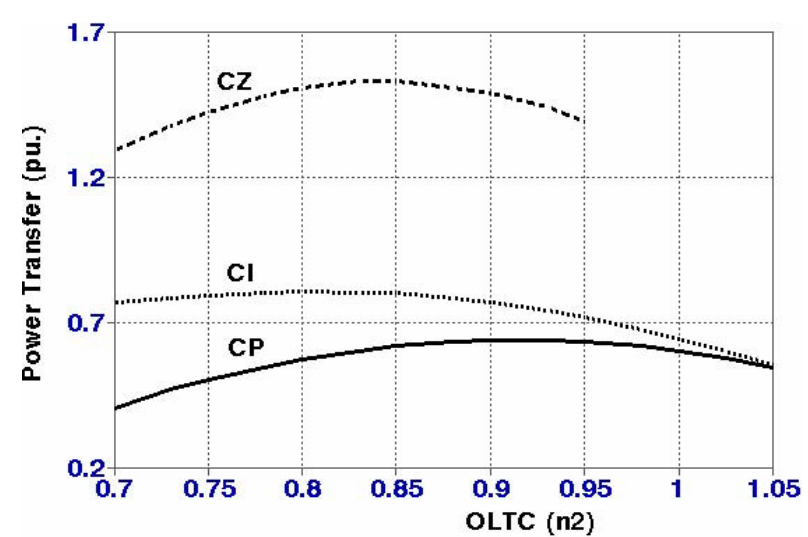

Fig. 11 Effect of OLTC (n2) on Power transfer to load 3

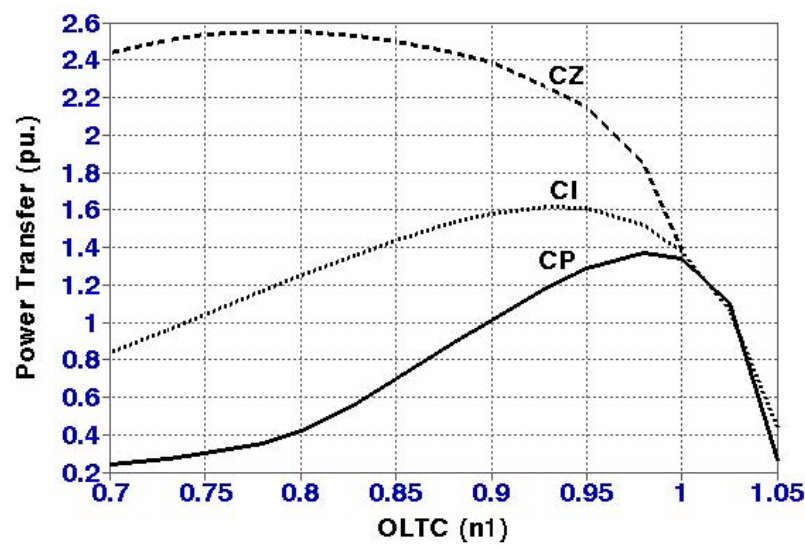

Fig. 12 Effect of OLTC (n1) on Power transfer to load 1

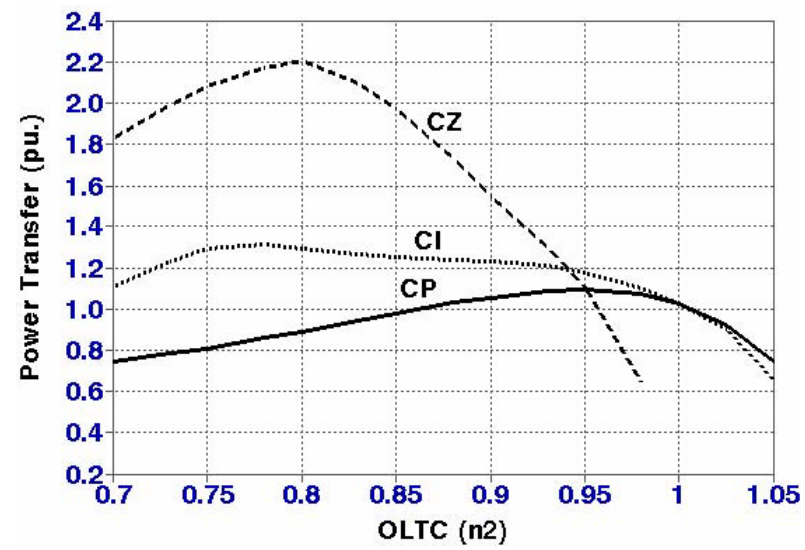

Fig. 13 Effect of OLTC (n2) on Power transfer to load 2

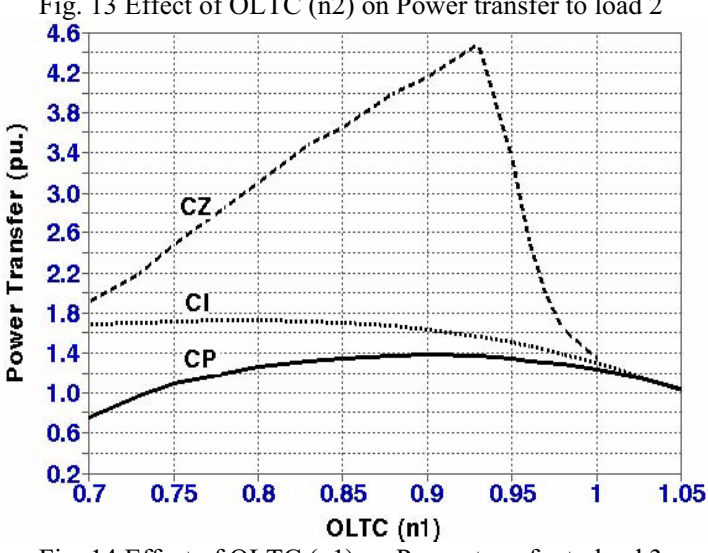

Fig. 14 Effect of OLTC (n1) on Power transfer to load 3

Moreover, it can be seen that before the reverse points are reached, the upward of OLTC operation increases the maximum power transfer to the load centre. Also these results give an indication that the maximum point of power transfer and thus the levels of these powers are different for different load models. It must be noted here that, in the constant impedance (CZ) load model, the nonlinear load behaviour is very sensitive to any system variation near to the static stability limit. That can be depicted from the sharp peaks indicated on figures 10 and 14. In case of constant power (CP) load model, the load power is constant however, the power transfer to the load bus through the OLTC branch is maximized and the other shares through other branches are varied.

\section{DETERMINATION OF OLTC SETTING USING ANN}

This section presents a novel approach for on-line determination of transformer OLTC optimal settings that corresponds to the maximum power transfer to the load using artificial neural network (ANN).

Topology of the ANN The topology of a multi-layer feedforward ANN consists of an input layer, a hidden layer and an output layer $[6,7]$.

Input layer Appropriate selection of input variables is the key to the success of the ANN application. Usually heuristic knowledge is required in choosing the appropriate variables. The power transfer from generation to the load centre is mainly concerned with the transformer OLTC settings. The optimal setting of the OLTC is affected by the load level. Therefore, these loading conditions are chosen as inputs to the ANN. These inputs are the load active and reactive power in addition to the generation active and reactive power at each bus in the system.

Hidden layer The computational power of the ANN increases with the addition of hidden layers. There are no general guidelines to determine the number of hidden layers and number of neurons per layer. Many applications have proved that ANN with single hidden layer posses sufficient capability of capturing complicated relations between input and output variables. In this paper, one hidden layer with five neurons was found satisfactory for estimating the optimal transformer OLTC settings.

Output layer Determination of the output layer is quite forward. Two output neurons were used for setting the OLTC of the two transformers. The unipolar sigmoid activation function was preferred. Appropriate scaling of the input and output variables were carried out in the $(0,1)$ range.

Training Scenario The scenario of the training process was started by initiating the ANN topology; i.e. the number of input nodes, the number of hidden layers, the number of neurons in different hidden layers, the number of neurons in the output layer, the type of hidden and output activation functions, and the number of presentation cycles (epochs). It must be noted, that, by adjusting the number of hidden layers and the number of simulated neurons within each layer, the performance of an ANN can be either enhanced or degraded. There are no general guidelines for a priori knowledge of 
which ANN architecture would perform the best for a given application. The researchers have experimented with different architecture's to find out the most suitable configuration.

Testing Scenario For validating the ANN performance the scenario of the testing process is started once the training process is completed and a set of new input variables are applied to the designed ANN. The proposed method has been applied to the system shown in Fig. 8. The obtained results for this scenario are recorded in Tables I, II and III. The three load models under consideration are tested on different operating conditions. The transformers turns ratios are varied one at a time. The results in Table I have been obtained for the constant power (CP) load model. The table shows that the maximum testing absolute errors are: 5.06\% (n1) \& 5.623\% (n2). Similarly, Tables II \& III show these errors for the constant current $(\mathrm{CI})$ and the constant impedance (CZ) load models, respectively. The maximum errors obtained in this case are: $6.0 \%(\mathrm{n} 1), 3.94 \%(\mathrm{n} 2)$ and $3.66 \%(\mathrm{n} 1), 3.49 \%(\mathrm{n} 2)$, respectively. Also tables present the average and the standard deviations for the three different load models. These deviations are: 1.092/1.731 (n1), 1.762/2.215 (n2) for CP load; 1.499/2.375 (n1), 1.275/1.636 (n2) for CI model; and 1.66/1.474 (n1), 0.825/1.19 (n2) for CZ model. From these results, it can be seen that the $\mathrm{CP}$ load and the $\mathrm{CI}$ load models have nearly the same error level. While, the CZ load model has the less error level. The ANN is efficient for the prediction of the optimal value of transformer turns ratio settings corresponding to the maximum power transfer to the load centre.

\section{CONCLUSIONS}

An efficient method for the on line prediction of transformer OLTC settings giving maximum power transfer to the load centre is analysed. The proposed technique has been tested on a Six-bus power system. Numerical results show that the operation of transformer OLTC has a major effect on the maximum power transfer and thus on the static stability margin. Load models have a great effect not only on the maximum power transfer but also on the optimal settings of transformer OLTCs. The Artificial neural network (ANN) proved to be an efficient tool for the prediction of the optimum value of transformer OLTC settings corresponding to the maximum power transfer.

\section{REFERENCES}

[1] T.X .Zhu, S.K. Tso. and K.L.Lo. "An Investigation into the OLTC Effects on Voltage Collapse". IEEE Transaction on power Systems vol.15. No.2, May, 2000, pp.515-521.

[2] C.W.Taylor,"Power System Voltage Stability".New York; McGrawHill, Inc., 1994.

[3] Les M.Hajagos, and Behnam Danai "Laboratory Measurements and Models of Modern Loads and Their Effects on voltage Stability Studies" IEEE Transaction on Power Systems, vol. 6, No.2 May, 1998, pp.584-591.

[4] Tamura, Y.Mori, H.Iwamoto,S. “ Relationship Between Voltage Instability and Multiple Load Flow Solutions in Electric Power Systems". IEEE Transaction on Power Apparatus and Systems, PAS102, No.5, 1993, pp.1115-1125.
[5] J.Qiu and S.M.Shahidelhpour;" A New Approach For Minimizing Power Losses and Improving Voltage Profile”. IEEE Transaction on Power Systems, Vol. PWRS-2, No.2, May 1987, pp. 287-295.

[6] M.A. EL-Sharkawi, R, J. Marks and Siri Weerasooriya " Neural Networks and Their Application to Power Engineering" control and Dynamics serial Academic Press, 1991.

[7] Qin Zhou et.al,."Application Of Artificial Neural Networks In Power System Security And Vulnerability Assessment". IEEE Transaction on Power Systems, Vol.9, No.1, February, 1994. pp. 525-532. 